\title{
Developmental setback in severe visual impairment
}

\author{
Hilary D Cass, Patricia M Sonksen, Helen R McConachie
}

\begin{abstract}
Developmental setback in children initially thought to be of normal cognitive potential is a serious complication of severe visual impairment; the prevalence, diagnostic specificity, clinical presentation, and factors that contribute to its genesis require systematic investigation. The findings are reported of a retrospective case review over a 15 year period of children attending the developmental vision clinic at the Wolfson Centre of the Institute of Child Health. One hundred and two children met the inclusion criteria of a period of normal development confirmed at initial assessment when aged less than 16 months, absence of additional disabilities, and follow up to at least 2.5 years of age.
\end{abstract}

Developmental setback in their second or third year occurred in $10(31 \%)$ of 32 children who were totally blind throughout (minimal perception of light or less), one (4\%) of 25 who, though blind at first assessment, showed visual improvement, and none of 49 children with better vision throughout (awareness for near, large objects). This represents a significantly greater risk for totally blind children than for the other groups. The course and characteristics of the affected children varied, but all had visual diagnoses involving the nervous elements of the visual system, and $60 \%$ had major social adversity factors. The role of primary maldevelopment of the central nervous system, the degree of visual impairment, the developmental and emotional climate, and the stage of attentional and behavioural development in the causation of adverse developmental outcome are discussed.

(Arch Dis Child 1994; 70: 192-196)

Adverse developmental outcome has long been recognised as a complication of severe visual impairment. Although many blind children are reported to have additional neurodevelopmental problems from birth, there are also reports of developmental setback in children initially thought to be of normal cognitive potential; this can be expressed in various forms including developmental stasis, regression, and autistic disorder. Following Kanner's paper on early infantile autism, ${ }^{1}$ Keeler described developmental stasis and 'autistic-like' behaviour in children blind due to retinopathy of prematurity. ${ }^{2}$ Fraiberg and

Freedman presented a number of detailed case studies drawing attention to the social withdrawal, lack of explorative play, and stereotyped behaviour observed in such children, and noted that this pattern of behaviour was not specific to children with retinopathy of prematurity, but occurred in up to $25 \%$ of children with a wide range of visual disorders. ${ }^{3}$ A similar pattern of developmental disorder was described by Norris et al in a study of 295 preschool children, of whom $29 \%$ were blind due to causes other than retinopathy of prematurity. ${ }^{4}$

Several workers have shown that poor neurodevelopmental outcome may be related to dysgenesis of the central nervous system. Dekaben, investigating a series of 13 patients with congenital retinal dystrophy, reported mental retardation in $10(77 \%)$ and a variety of neurological complications such as epilepsy and cerebral palsy in eight $(62 \%) .{ }^{5}$ In a study of 40 children with optic nerve hypoplasia, Roberts-Harry et al showed computed tomogram abnormalities in $27(67 \%)$ and neurological abnormalities in $22(55 \%){ }^{6}$ Clearly the nature, site, and extent of any cellular dysgenesis will vary between and within visual diagnostic groups. For example, abnormalities of the cerebellar vermis were reported by Nickel and Hoyt in $10 \%$ of patients with Leber's amaurosis, ${ }^{7}$ and are a characteristic feature of Joubert's syndrome. In addition to the midline abnormalities expected in a group with optic nerve hypoplasia, Roberts-Harry et al identified several children with cortical atrophy and porencephaly; they noted that disease severity and scan abnormalities were good, but not absolute, predictors of neurological impairment. ${ }^{6}$ Similarly, Vaizey et al found a correlation between the severity of visual impairment and the incidence of neurodevelopmental delay in a series of 30 patients with congenital retinal dystrophies. ${ }^{8}$ Black and Sonksen showed that children with congenital retinal dystrophies complicated by central nervous system malformations or additional medical problems are more likely to have a learning disability. ${ }^{9}$

These studies raise the question of whether poor developmental outcome is wholly attributable to the underlying neuropathology or whether it is at least partially attributable to other factors. Prenatal influences clearly play a major part where development is delayed from the outset. The situation is more complex in children who undergo developmental setback after a period of apparently normal development. The latter phenomenon is well recognised in sighted children with a range of disorders including autism, ${ }^{10}$ Landau-Kleffner syndrome, and a number of other epileptic
Correspondence to: Dr Hilary Cass, Harper House Children's Service, WD7 7HU.

Accepted 5 December 1993 
Table 1 Distribution of visual diagnoses within visual impairment groups

\begin{tabular}{llllr}
\hline & $\begin{array}{l}\text { Group 1 } \\
\text { (blind } \\
\text { throughout) }\end{array}$ & $\begin{array}{l}\text { Group 2 } \\
\text { (vision } \\
\text { improved) }\end{array}$ & $\begin{array}{l}\text { Group 3 } \\
\text { (better vision } \\
\text { throughout) }\end{array}$ & Total \\
\hline Diagnosis & 1 & - & 5 & 6 \\
Cortical & - & 1 & 2 & 3 \\
Optic atrophy & 2 & 3 & 1 & 6 \\
Optic nerve hypoplasia/septo-optic dysplasia & - & - & 1 & 1 \\
Optic nerve colobomata & 5 & 3 & 1 & 9 \\
Retinopathy of prematurity & 8 & - & - & 8 \\
Norrie's disease & 1 & 1 & 1 & 15 \\
Other retinal dysplasias & 8 & 2 & 5 & 2 \\
Retinal dystrophy, including Leber's amaurosis & - & - & 2 & 5 \\
Choreoretinitis & - & - & 5 & 2 \\
Oculocutaneous albinisim & 5 & 4 & 7 & 16 \\
Microphthalmia/anophthalmia & - & - & 2 & 2 \\
Colobomata & - & - & 1 & 2 \\
Aniridia & - & 1 & 1 & 4 \\
Buphthalmos & - & 5 & 12 & 102 \\
Cataracts & - & - & 2 & \\
Eye movement disorders & 2 & 1 & 1 & 2 \\
Miscellaneous & 32 & 21 & 49 & \\
Total & & & & 2 \\
\hline
\end{tabular}

syndromes. Our clinical experience with severely visually impaired children suggests that it also occurs in this group, though the balance of factors triggering its onset may be different. We have therefore undertaken a retrospective study of the developmental progress of severely visually impaired children to document the characteristics of those patients who underwent developmental setback after a period of normal development.

\section{Subjects and methods}

The study group was drawn from 615 severely visually impaired children who had been seen during a 15 year period in the developmental vision clinic at the Wolfson Centre of the Institute of Child Health. The focus of the clinic is developmental management. Children attending the clinic are reviewed at intervals of four to 12 months depending on the age of the child, with younger children being seen more often to facilitate regular updating of developmental guidance. They are comprehensively assessed at each visit by a multidisciplinary team experienced in the medical and developmental management of infants and young children with severe visual disability.

The criteria for inclusion were age less than 16 months when first seen, normal development at first assessment with no additional disabilities, and follow up to at least 2.5 years of age. This gave a study population of 102 children whose case records were analysed retrospectively. The children were divided into three groups according to the degree of visual impairment over time. Group 1 had minimal or no perception of light throughout; group 2 had this level of vision at the outset, but improved at least to a level where they could see a 5 inch spinning ball within 10 inches, and group 3 had vision which was at this better level from the outset. Table 1 gives the distribution of visual diagnoses between the three groups.

Cognitive progress was monitored using the Reynell-Zinkin scales of mental development for visually impaired children. ${ }^{11}$ These scales provide an age equivalent level of function on each subscale and are standardised for blind, partially sighted, and sighted children. The three most intellectually loaded subscales - sensorimotor understanding (non-verbal cognition), verbal comprehension, and expressive language - were used. In addition, concerns expressed by either the parents or assessor about the child's social development or behavioural status, or both, were noted, as were details of any adverse social factors.

Setback was defined as stasis or regression on one or more subscales sustained over at least two consecutive assessments, or increasing disorder in social interaction and communication, or both.

\section{Results}

Eleven (11\%) children underwent developmental setback; $10(31 \%)$ of the 32 children who were totally blind throughout compared with only one $(5 \%)$ of the 21 whose vision improved, and none of the 49 with better vision throughout. Fisher's test of exact probability showed statistically significant relationships between visual status and developmental outcome; children blind throughout were at greater risk than those whose vision improved and at substantially greater risk than those with better vision throughout $(p<0.05$ and $\mathrm{p}<0.0001$ respectively).

Table 2 gives the background details and course of those children who underwent developmental setback. Interestingly, all the children whose visual disorder was peripheral in origin, such as buphthalmos or cataracts, had normal developmental outcome, whereas the group with developmental setback all had visual disorders which affected the nervous elements of the visual system. Furthermore, two of the seven children with anophthalmia deteriorated, whereas all nine of the children

Table 2 Background details and course of children undergoing developmental setback

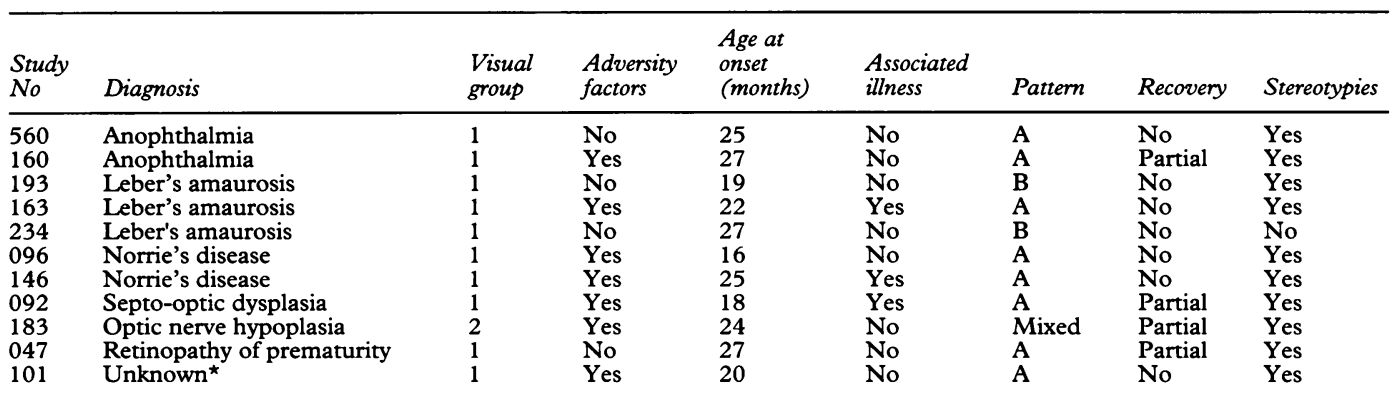

^Aetiology of visual impairment not determined, but peripheral causes excluded. 
with microphthalmia, representing the less severe end of the globe dysgenesis spectrum, had a normal outcome.

Factors potentially detrimental to developmental climate were compared for children with normal outcome and those with developmental setback within group 1 . These included prolonged admissions to hospitals, social problems such as marital discord or breakdown, maternal depression, parental drug addiction, major financial or housing problems, or a combination of these factors. Such factors were documented in $23 \%$ of children who had normal outcome compared with $60 \%$ of children who had developmental setback, representing a statistically significant excess of adversity factors in the latter group $(p<0.05$, Fisher's test of exact probability).

The age at onset of setback was between 16 and 27 months, with three of the children having a physical illness before onset. An encephalopathic process, the cause of which was never established despite extensive investigation, was suspected in two of the three. The first (study No 163) had a non-specific illness at $2 \cdot 3$ years with lethargy, submandibular swellings, and anorexia, after which she became introspective, withdrawn, and reluctant to walk; electroencephalography (EEG) showed mild hemispheric asymmetry only. She subsequently died unexpectedly at the age of 3.7 years. The second child (study No 092) underwent developmental setback over a two month period from 18 months of age, during which she was pale, fretful, and irritable; EEG showed excess slow wave activity in the temporal and Sylvian regions with poverty of paracentral activity. This was followed by episodes of vomiting and malaise at intervals thereafter and a hypoglycaemic collapse at 4.2 years, though cortisol levels were normal. Growth hormone deficiency was shown two months later. The third child (study No 146) did not have an acute illness but spent 29 days in hospital during his second year for treatment of intractable eczema, a scalp abscess, and an ophthalmic operation.
A Sensorimotor understanding

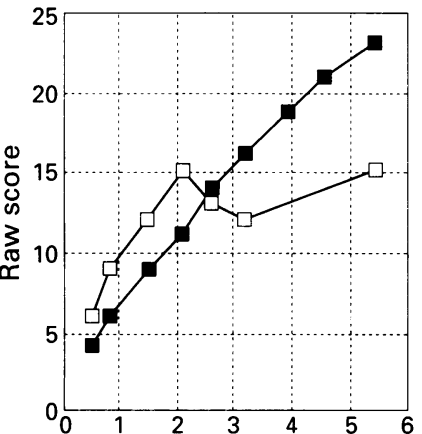

B

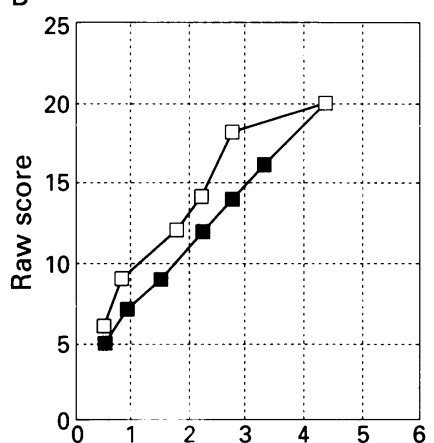

C

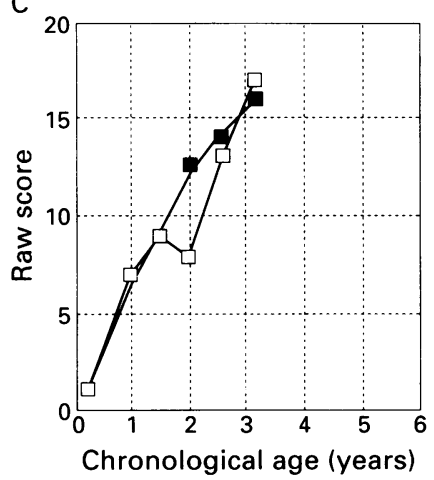

\section{$\neg \square$ Patient $\rightarrow-$ Blind mean}
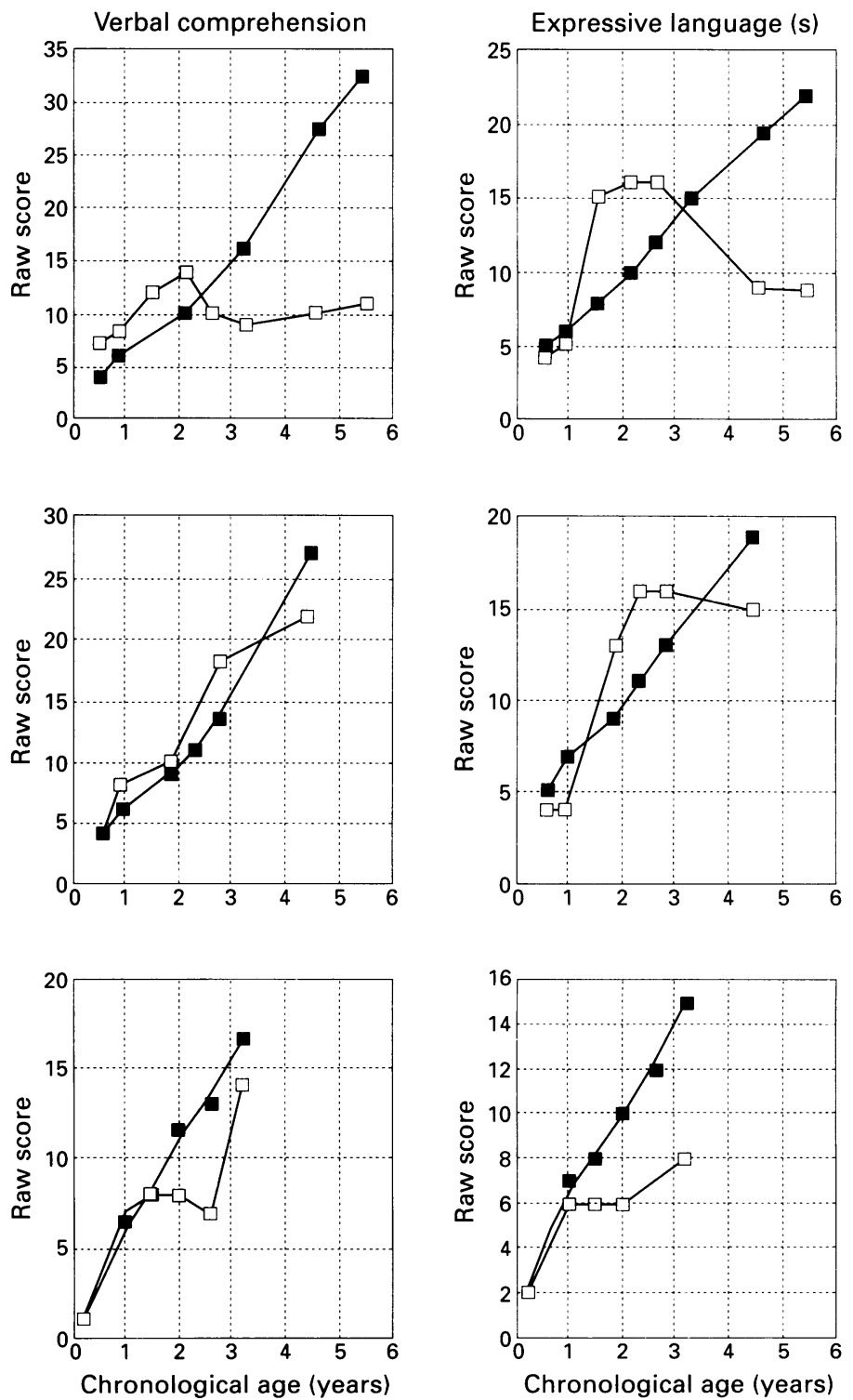

Patterns of deterioration and recovery. (A) Pattern A; (B) pattern B; and (C) partial recovery. 
The course and characteristics of the setback in the affected children were not homogeneous. Two broad patterns emerged, with varying degrees of change in social accessibility and behaviour being central features of both; in pattern A (illustrated in fig A) developmental stasis or regression occurred in association with a loss of explorative and manipulative play, whereas in pattern $B$ (illustrated in fig B) there was increasingly disordered social communication with continuing acquisition of non-verbal cognitive skills. Stereotypies including eye poking, rocking, flapping, and face tapping were features of both patterns of deterioration with the examiner often noting that the stereotypies appeared to be blocking learning. Partial recovery occurred in four children, apparently in association with changes in the developmental environment such as additional developmental support in the home or appropriate nursery school placement. In three of these four children, however, the recovery in non-verbal cognition was more complete than the recovery in expressive language with the children showing continuing disorder rather than delay in their communication skills (fig $\mathrm{C}$ ).

\section{Discussion}

This study highlights the fact that developmental setback is a significant clinical problem among children with severe visual impairment, and is most prevalent in those with the greatest degree of visual impairment. The range of visual diagnoses and varied presentations of the affected children suggests that the phenomenon is clinically and pathogenically heterogeneous. Common to all the affected patients is the relatively narrow age range during which the onset of developmental stasis or disorder occurs. We postulate that the interaction of inherent neurological susceptibility with an adverse environmental climate at a critical stage of behavioural development results in the onset of developmental impairment.

The importance of the degree of visual impairment in this equation has been discussed previously. For example, Keeler compared the incidence of developmental arrest and autistic-like behaviour in three groups of children in which there was no overlap of visual diagnosis; those children who were totally blind due to retinopathy of prematurity were at greater risk than a group of congenitally blind children with less severe visual impairment, whereas a third group of children, blinded postnatally, seemed to be relatively protected. ${ }^{2}$ Because the visual diagnoses that result in greater degrees of visual impairment are those known to be associated with central nervous system disorder, ${ }^{5-8}$ however, it is difficult to separate the relative contribution of these factors to the pathogenesis of developmental setback. In our study, though some visual disorders were represented in all three groups (table 1), others, by definition, could not be represented in the less severely visually impaired groups - for example, Norrie's disease. Even for those visual disorders that were represented in all three groups it may be that the more visually impaired children were also the more neurologically damaged, and therefore most at risk developmentally. Further neuroimaging studies are required to evaluate the frequency, type, and extent of central nervous system dysgenesis in blind children with normal development, as well as in those with an adverse developmental outcome.

An alternative perspective of the role of vision is provided by Goodman and Ashby ${ }^{12}$ who describe three boys with delayed visual maturation and autistic impairment who made striking progress in social interaction, language, and play after an improvement in functional vision. In this context, a group 2 child with Leber's amaurosis who did not fulfil the criteria for developmental setback is of interest; she initially had perception of light only and her development progressed steadily just below the mean for blind children on all Reynell-Zinkin subscales. At the age of 20 months her parents and the assessor expressed concern that her social interactions and behaviour were becoming disordered. At 25 months her vision had improved to a level where she was able to see a $2 \cdot 5$ inch ball within 12 inches. Coincident with this her behaviour and social interaction reverted to normal, and her eventual outcome was normal. It is possible in this patient, and in the patients described by Goodman and Ashby, ${ }^{12}$ that improved vision at a critical time may have protected against irreversible developmental impairment.

Attention has previously been drawn to the influence of adverse social factors in developmental setback. ${ }^{2-4}$ Although in our retrospective study $64 \%$ of the affected children had experienced such factors, this needs to be interpreted with caution as there may have been a reporting bias in children about whom there was already developmental concern. More objective data are required on developmental and social climate in good and poor outcome, as the optimum conditions for visually impaired children may differ from those described for sighted children (V Moore, $\mathrm{H} \mathrm{McC}$ Conachie, unpublished data).

The importance of encephalitis in the genesis of developmental setback is not clear; it was not reported as a complication in a Finnish study reviewing 73 children presenting with encephalitis over a 10 year period. ${ }^{13}$ DeLong et al, however, described acquired reversible autistic syndrome after acute encephalopathic illness in three children ranging from 5 to 11 years of age. ${ }^{14}$

Within the first three years of life there appears to be a vulnerability to developmental setback with autistic withdrawal, even in the absence of acute physical illness. This is described in children with disorders in which biological predetermination is implicit, such as Rett's syndrome ${ }^{15}$ and tuberous sclerosis, ${ }^{16}$ as well as in children without overt preceding neuropathology. ${ }^{10}$ The stage in the development of attention control and behavioural 
independence which prevails in the second half of the second year may constitute a further risk factor in our group. At this age children maintain the focus of their attention inflexibly by blocking out other stimuli and interactions; given the lack of a visual channel the blind child's attention is even more difficult to redirect. In addition, blind children may have delay in their ability to physically explore the environment. ${ }^{17}$ Self stimulation with stereotypies such as eye poking or rocking are common, and it is not difficult to appreciate the ease with which such behaviours can become self reinforcing to the exclusion of other inputs.

The two patterns of deterioration observed in our study parallel those described by other workers. The more cognitively impaired children with pattern A developmental profiles were similar to those described previously. ${ }^{2-4}$ Pattern B profiles were only observed in two children, both of whom had Leber's amaurosis. They appeared to follow the same clinical course as that described by Rogers and Newhart-Larson in five children with Leber's amaurosis. ${ }^{18}$ Further studies are needed to clarify whether this pattern is specific to Leber's amaurosis.

There is a lack of consistency in published work as to whether the affected children are correctly described as falling within the autistic continuum. Certainly, the children who underwent developmental setback in this study all showed disorders of social interaction, communication, and imagination in association with a restricted and repetitive repertoire of activities. Some caution is required in making a diagnosis of autism, however, because impairments in all these features must be evaluated in relation to normally developing blind children rather than sighted normal subjects. As yet there are no standardised data on the normal development of social relatedness in blind children, and many of the important features used for diagnosis in sighted children, such as the establishment of joint attention and the ability to turn-take, are much harder to evaluate in the visually impaired. Standardised rating tools such as the Childhood Autism Rating Scale ${ }^{19}$ and the Autism Behaviour Checklist $^{20}$ have been used by previous workers. ${ }^{18}$ In the absence of standardisation of these tools on children with uncomplicated visual impairment it is not possible to assess the extent to which features within the range of normality for visually impaired children - for example, 'blindisms' such as eye poking, or language features such as pronoun reversal, might weight the results and reduce the discriminatory value of the test. It is therefore essential that data are collected on normal social and behavioural development in children with severe visual impairment so that criteria for disorders in this group can be clarified.

Further investigation is necessary to delineate the relative contribution to developmental setback of the factors highlighted by this study. If some of these are found to be reversible, the early detection of children at risk of developmental setback should create opportunities for remediation. In addition, the identification of a group in which the evolution of impairments in social communication can be observed prospectively may provide a valuable model for developing our understanding of the neurological substrate of autistic symptomatology.

1 Kanner L. Autistic disturbances of affective contact. Nervous Child 1943; 2: 217-50.

2 Keeler WR. Autistic patterns and defective communication in blind children with retrolental fibroplasia. In: Hoch PH, Zubin J, eds. Psychopathology of communication. New YH, Zubin J, eds. Psychopathology of comm

3 Fraiberg S, Freedman D. Studies in the ego development of the congenitally blind. Psychoanalytic Study of the Child 1964; 19: 113-69.

4 Norris M, Spaulding P, Brodie F. Blindness in children. Chicago: University of Chicago Press, 1957.

5 Dekaben AS. Mental retardation and neurological involvement in patients with congenital retinal blindness. Dev Med Child Neurol 1972; 14: 436-44.

6 Roberts-Harry J, Green SH, Willshaw HE. Optic nerve hypoplasia: associations and management. Arch Dis Child 1990; 65: 103-6.

7 Nickel B, Hoyt CS. Leber's congenital amaurosis. Is mental retardation a frequent associated defect? Arch Ophthalmol 1982; 100: 1089-92.

8 Vaizey MJ, Sanders MD, Wybar KC, Wilson J Neurological abnormalities in congenital amaurosis of Leber. Arch Dis Child 1977; 52: 399-402.

9 Black MM, Sonksen PM. Congenital retinal dystrophies: a study of early cognitive and visual development. Arch Dis Child 1992; 67: 262-5.

10 Hoshino Y, Kaneko M, Yashima Y, Kumashiro H, Volkmar FR, Cohen DJ. Clinical features of autistic children with setback course in their infancy. $f_{p n} \mathcal{f}$ Psychiatry Neurol 1987; 41: $237-46$.

11 Reynell J. Manual for the Reynell-Zinkin scales. London: National Foundation of Education Research, 1979.

12 Goodman R, Ashby L. Delayed visual maturation and autism. Dev Med Child Neurol 1990; 32: 814-9.

13 Rantala $H$, Uhari $M$, Uhari $M$, Saukkonen A, Sorri $M$. Outcome after childhood encephalitis. Dev Med Child Neurol 1991; 33: 858-67.

14 DeLong GR, Bean SC, Brown FR. Acquired reversible autistic syndrome in acute encephalopathic illness in autistic syndrome in acute encephal
children. Arch Neurol 1981; 38: 191-4.

15 Hagberg B, Aicardi J, Dias K, Ramos O. A progressive syndrome of autism, dementia, ataxia and loss of purposeful hand use in girls: Rett's syndrome: report of 35 cases. Ann Neurol 1983; 14: 471-9.

16 Gomez MR, ed. Tuberous sclerosis. New York: Raven Press, 1988: 28-32.

17 Fraiberg S. Insights from the blind. London: Souvenir Press, 1977.

18 Rogers SJ, Newhart-Larson S. Characteristic of infantile autism in five children with Leber's amaurosis. Dev Med autism in five children with Leber

19 Schopler E, Reichler R, DeVillis R, Daly K. Towards an objective classification of childhood autism: childhood autism rating scale (CARS). $\mathcal{F}$ Autism Dev Disord 1980 10: $91-103$.

20 Krug D, Arlick J, Almond P. Behaviour checklist for identifying severely handicapped individuals with high levels of autistic behaviour. $f$ Child Psychol Psychiatry 1980; 21: 221-9. 